\title{
Ferromagnetic one dimensional Ti atomic chain
}

\author{
Jisang Hong \\ Department of Physics, Pukyong National University, Busan 608-737, Korea
}

\begin{abstract}
Using the full potential linearized augmented plane wave (FLAPW) method, we have explored the magnetic properties of one dimensional (1D) Ti atomic chain. Astonishingly, we for the first time observed that the 1D Ti atomic chain has ferromagnetic ground state even on $\mathrm{NiAl}(110)$ surface although the Ti has no magnetic moment in bulk or macroscopic state. It was found that the physical property of direct exchange interaction among Ti atoms occurred in free standing state is well preserved on $\mathrm{NiAl}(110)$ surface and this feature has an essential role in ferromagnetism of $1 \mathrm{D} \mathrm{Ti}$ atomic chain. It was shown that the $\mathrm{m}=|2|$ state has the largest contribution to the magnetic moment of $\mathrm{Ti}$ atom grown on $\mathrm{NiAl}(110)$ surface. In addition, we found that the magnetic dipole interaction is a key factor in the study of magnetic anisotropy, not the magnetocrystalline anisotropy arising from spin-orbit interaction.
\end{abstract}

Key words: 1D Ti atomic chain, Ferromagnetic wire, Magnetic anisotropy, FLAPW

$P A C S$ :

\section{Introduction}

It is well known that ultra small nano scale materials display many peculiar phenomena not observed in macroscopic state and the study of nano scale material has become the most interesting issue. These days, due to remarkable progress in the field of atomic manipulation technique one can even build 1D nanosystems. For instance, 1D Co ferromagnetic (FM) nanowire was grown on $\operatorname{Pt}(997)$ step surface and a magnetic anisotropy energy was measured [1]. Also, 1D Au chains through scanning tunneling microscopy tip control were prepared on $\mathrm{NiAl}(110)$ surface and the electronic structures were investigated [2]. In addition, a giant magnetic anisotropy was obtained in single Co atom and nanoparticles [3]. Overall, the ultra small materials display many exciting 
physical properties and a great amount of research effort has been focused on these materials.

Especially, if the spin degree of freedom is included one can expect more rich physical phenomena. Indeed, one may easily obtain many interesting experimental data extracted from nano magnetic materials since the extensive progress has been achieved in the filed of nano magnetism. However, the fully quantitative theoretical investigations are still in early stage. In the theoretical view point for magnetic properties of 1D nanostructures, we already presented the results of magnetic anisotropy calculations of infinite Co nano chains and interesting size dependence of magnetic properties in finite Co nano wires $[4,5]$. It is well understood that most of physical properties of materials are very sensitive to the change in underlying electronic structure and particularly in ultra small size the effect will be very significant. Therefore, we can anticipate that the ultra small nano scale materials show unexpected physical properties different from those observed in bulk or macroscopic states such as half metallic character in suspended Co nanowires [6].

In one dimensional geometry the electronic structure will be substantially altered due to the reduction of dimension and several theoretical investigations were performed to explore the peculiar magnetic properties of $1 \mathrm{D}$ nanostructures. For instance, it was theoretically presented that a Pd atom in unsupported one dimensional nanowire structure has magnetic moment [7]. On the other hand, it was shown that three dimensional nanowires of non-magnetic (NM) simple metals, for instance $\mathrm{Na}, \mathrm{Cs}$, and $\mathrm{Al}$, have ferromagnetic ground state at certain critical radii although the calculations are based on rather primitive model [8]. It is quite clear that $\mathrm{Cu}$ is non-magnetic material in bulk state. But, very recent quantum conductance measurement indicated that pure 1D $\mathrm{Cu}$ nano wire has spin polarized electronic band structure due to oxygen effect $[9,10]$ and our theoretical calculations revealed that the pure $1 \mathrm{D} \mathrm{Cu}$ atomic chain has no magnetic moment for various inter-atomic distances. But, very surprisingly it was demonstrated that the spin polarization can arise in $1 \mathrm{D} \mathrm{CuO}$ diatomic nanowire when the inter-atomic distance between $\mathrm{Cu}$ and $\mathrm{O}$ is larger than equilibrium distance [11]. These theoretical and experimental results imply that the reduction of dimension may even affect the magnetic property of material.

So far, most of experimental data found in nano magnetic systems the sample materials were basically magnetic in bulk or macroscopic state and simply the size of material was greatly reduced. Neither experimentally nor theoretically investigated the possibility of achieving magnetic state in purely $1 \mathrm{D}$ atomic scale from non-magnetic material in macroscopic size. But, as mentioned above the electronic structure of ultra small material in low dimension is quite different from the one found in bulk or macroscopic state. Therefore, it will be of interest to explore whether or not we can find magnetic moment in reduced 
dimension through fully quantitative calculations, whereas the material is nonmagnetic in bulk state. In this spirit, we considered well known non-magnetic element $\mathrm{Ti}$ and investigated the existence of ferromagnetic ground state in one dimensional atomic chain structure.

\section{Numerical Methods}

The full potential linearized augmented plane (FLAPW) method was employed in our calculations. Therefore, no shape approximation is assumed in charge, potential, and wavefunction expansions. We treat the core electrons fully relativistically. The generalized gradient approximation was employed to describe exchange correlation [12]. Spherical harmonics with $l_{\max }=8$ were used to expand the charge, potential, and wavefunctions in the muffin tin region. Energy cut offs of $225 \mathrm{Ry}$ and 13.69 Ry were implemented for the plane wave star function and basis expansions in the interstitial region. The $\mathrm{NiAl}(110)$ substrate was simulated by 7 layers of $\mathrm{NiAl}(110)$ slab. The $1 \mathrm{D}$ atomic chain is placed along the $x$ axis. We impose periodic boundary condition along the lateral direction ( $y$ axis) for computational purpose. The lattice constants of $a=5.454$ a.u. and $b=15.425$ a.u. were used for $\mathrm{NiAl}(110)$ substrate. The same lattice parameters were assumed for the calculations of free standing nanowire. When we check the possibility of antiferromagnetic ordering in the 1D Ti nanowire, the size of unit cell is increased twice in the x-direction.

\section{Results and Discussions}

First, we explored the existence of magnetic phase in unsupported Ti nanowire with the inter-atomic distance of $\mathrm{NiAl}(110)$ surface as shown in above. Very surprisingly, it was obtained that the total energy of ferromagnetic phase in free standing 1D Ti nanowire is lower than that of non-magnetic state. The energy gain resulting from the spin polarization was $539 \mathrm{meV} /$ atom. In addition, the calculated magnetic moment of Ti atom was $1.88 \mu_{B}$. Based on this promising result, we performed more realistic calculations, i.e. the $1 \mathrm{D}$ $\mathrm{Ti}$ atomic chain grown on $\mathrm{NiAl}(110)$ surface. In Fig. 1, the structure of unit cell considered in our calculations is schematically illustrated. The physical property of nanoscale material is sensitive to underlying electronic structure and the electronic structure is dependent on atomic structure of material. It is therefore important to achieve well optimized atomic positions for quantitative calculations. The optimized atomic structure was found with FLAPW calculations guided by force and energy minimization procedures. In Table 1 , the vertical positions of $\mathrm{Ti}$ and substrate atoms are presented. As one can 
see, the surface layer shows buckling state and this behavior in $\mathrm{NiAl}(110)$ surface is experimentally observed [2]. Nonetheless, one can see that the subsurface layers are very flat. Now, the main issues is to investigate if the $1 \mathrm{D} \mathrm{Ti}$

Table 1

Vertical positions of Ti and substrate atoms in atomic unit.

\begin{tabular}{lccccccc}
\hline atom & & $\mathrm{Ni}$ & & & $\mathrm{Al}$ & $\mathrm{Ti}$ \\
\hline type & 1 & 2 & 3 & 1 & 2 & 3 & \\
distance & 11.598 & 11.508 & 7.712 & 11.708 & 7.712 & 7.712 & 15.504 \\
\hline
\end{tabular}

nanowire can maintain ferromagnetic phase in ground state. For this purpose, we consider the 1D Ti nanowire on $\mathrm{NiAl}(110)$ for NM, FM, and antiferromagnetic (AFM) states, respectively and calculate their total energies. For convenience, the total energy of FM state is given as zero for reference. The calculated results are presented in Table II. We found that the energy gain due to spin polarization from $\mathrm{NM}$ to $\mathrm{FM}$ state is $245 \mathrm{meV} / \mathrm{cell}$ and furthermore it was obtained that the FM state is more stable than AFM state. Overall, we conclude that the 1D Ti atomic chain has ferromagnetic ground state on $\mathrm{NiAl}(110)$ surface. The calculated magnetic moment of Ti atom was $1.24 \mu_{B}$, whereas no induced magnetic moment was observed in any other atoms in substrate. To the best of our knowledge neither experimentally nor theoretically reported the existence of ferromagnetic ground state in 1D Ti nanowire so far. Consequently, we believe that our theoretical calculations are the first prediction that one may observe ferromagnetic phase in 1D Ti atomic chain grown $\mathrm{NiAl}(110)$ surface.

Table 2

Total energies for 1D Ti/NiAl(110) in $\mathrm{meV} /$ cell.

\begin{tabular}{cccc}
\hline & NM & FM & AFM \\
\hline Energy & 245 & 0 & 200 \\
\hline
\end{tabular}

Two physical mechanisms can account for the ferromagnetism in 1D Ti atomic chain; direct exchange interaction among $\mathrm{Ti}$ atoms and indirect one mediated through substrate electrons. It is trivial that the direct exchange interaction among $\mathrm{Ti}$ atoms is the origin of ferromagnetism for unsupported nanowire. When the Ti chain is grown on certain substrate, one should take into account the effect of indirect exchange interaction through substrate materials. Indeed, the importance of indirect exchange interaction in two dimensional $\mathrm{Mn}$ monolayer on $\mathrm{Nb}(001)$ surface was discussed in our theoretical calculations [13]. In the present system we observed that the energy gain due to spin polarization is substantially reduced compared to that found in free standing structure and this suppression is resulting from hybridization with substrate atoms, but there was no meaningful induced magnetic moment in surface $\mathrm{Ni}$ and $\mathrm{Al}$ atoms. Nonetheless, the ferromagnetic ground state is still maintained 
even on the $\mathrm{NiAl}(110)$ surface. We further analyzed the changes in charge transfer of Ti atom occurred in muffin tin (MT) region and found no meaningful disparity in the total number of charges for Ti atom in both NM and FM states (for FM state, the sum of majority and minority spin electrons in both free standing and on $\mathrm{NiAl}(110)$ ). The only modification realized in $\mathrm{Ti} / \mathrm{NiAl}(110)$ structure is the charge transfer about 0.3 electrons from majority to minority spin state and the spin polarization is originated from the spin splitting in d-state. We therefore believe that the direct exchange interaction is a key factor in ferromagnetism of $1 \mathrm{D}$ Ti nanowire although the magnitude of exchange interaction is affected by substrate atoms.

Since it was shown that the 1D Ti nanowire has ferromagnetic ground state, it is necessary to explore the density of states (DOS) features. We present the DOS of Ti adatom for free state and on $\mathrm{NiAl}(110)$ surface in Figs. 2(a) and 2(b), respectively. As shown in Fig. 2(a) for unsupported nanowire, one can see that there are no available states for minority spin electrons at the Fermi level and this indicates that the 1D Ti atomic chain is half metallic. In $\mathrm{Ti} / \mathrm{NiAl}(110)$ system, we found broadening of DOS due to hybridization with substrate elements and the half metallic feature is disappeared. It is clearly displayed that the d holes in majority states are increased, while the minority spin holes are decreased. This can nicely account for the suppression of magnetic moment in $\mathrm{Ti} / \mathrm{NiAl}(110)$. To provide a comprehensive picture for the peculiar ferromagnetism of $1 \mathrm{D}$ Ti atomic chain, we calculated the mresolved DOS. Figs. 3(a) and 3(b) show the m-resolved DOS of Ti for free standing and on $\mathrm{NiAl}(110)$ surface, respectively. The rough estimation reveals that the number of electrons below the Fermi level of $|m|=2,|m|=1$, and $|m|=0$ states are $0.76,0.74$, and 0.35 for unsupported case, respectively. This implies that the spin polarizations of $|m|=1$ and $|m|=2$ states play an important role for the ferromagnetism of free standing Ti nanowire. Substantial changes in m-resolved DOS of $\mathrm{Ti}$ were found on $\mathrm{NiAl}(110)$. The half metallic behavior observed in free standing structure is disappeared and one can find sizable minority spin states at the Fermi level instead. This stems from $|m|=1$ and $|m|=2$ orbitals, while the $\mathrm{m}=0$ state, i.e. $d_{3 z^{2}-r^{2}}$ orbital, does not play any role. The minority spin electrons mainly reside in $|m|=2$ states, i.e. $d_{x^{2}-y^{2}}$ and $d_{x y}$. Our calculations show that the number of minority spin electrons below the Fermi level in $|m|=2$ state are about 0.1 , whereas 0.18 electrons are in $|m|=1$ orbitals. In the majority spin band major change was seen in $|m|=1$ state, whereas there was no meaningful modification found in other two states like in unsupported structure. The m-resolved DOS of Ti/NiAl(110) presents that the net magnetic moments originated from $|m|=2,|m|=1$, and $|m|=0$ states are approximately $0.58,0.36$, and $0.25 \mu_{B}$, respectively. We observed that the charge transfer due to the hybridization effect is mainly occurred in $d_{x z}$ and $d_{y z}$ states and the $d_{3 z^{2}-r^{2}}$ state is almost intact even in the presence of substrate. Consequently, we conclude that the $d_{x^{2}-y^{2}}$ and $d_{x y}$ states are most important in ferromagnetism of $1 \mathrm{D} \mathrm{Ti}$ atomic chain on $\mathrm{NiAl}(110)$, whereas $|m|=0$ and 
$|m|=1$ orbitals manifest almost the same contribution to the ferromagnetism, but less important in ferromagnetism.

We now present the results of magnetic anisotropy of 1D Ti nanowires. As well known, two physical origins can account for the magnetic anisotropy of materials; magnetic dipolar interaction, so called shape anisotropy and magnetocrystalline anisotropy (MCA) due to spin-orbit interaction. It is straightforward to calculate the shape anisotropy and the magnetic dipolar interaction always prefers magnetization along the chain axis in 1D structure. The MCA arising from the spin-orbit interaction requires very accurate method for numerical calculations since the MCA is very sensitive to change in the underlying electronic structure. In our calculations the spin-orbit interaction is treated in second variational approach based on the ground state properties obtained from semi-relativistic calculations [14] and we used torque method [15] for the calculation of magnetic anisotropy. Since the wire is placed along the x-axis, we can write the expression of MAE as

$$
E=E_{0}-\sin ^{2} \theta\left(E_{1}+E_{2} \cos ^{2} \phi\right)
$$

where $\theta$ is a polar angle measured from the chain axis and $\phi$ is an azimuthal angle measured from y-axis. Then, one can easily obtain the relations $E_{x}-$ $E_{z}=E_{1}, E_{y}-E_{z}=-E_{2}$, and $E_{x}-E_{y}=E_{1}+E_{2}$. We then directly investigate $E_{1}$ and $E_{2}$ via torque method. It was found that $E_{1}=13 \mu \mathrm{eV}$ and $E_{2}=-20$ $\mu \mathrm{eV}$, so the direction of magnetization due to the spin orbit interaction is perpendicular to the chain axis (surface normal in our geometry). On the other hand we found that the shape anisotropy due to magnetic dipolar interaction is $33 \mu \mathrm{eV}$ and the direction of magnetization is along $\mathrm{x}$-axis as mentioned. Overall, we conclude that the direction of magnetization of 1D Ti nanowire is in $\mathrm{X}-\mathrm{z}$ plane and its angle is $21^{\circ}$ with respect to $\mathrm{x}$-axis

In summary, we for the first time theoretically investigated the existence of magnetic phase in 1D $\mathrm{Ti}$ atomic chain. It was found that free standing $\mathrm{Ti}$ nanowire has magnetic moment of $1.88 \mu_{B}$ and very interestingly the magnetic moment of $\mathrm{Ti}$ atom was still maintained even on the $\mathrm{NiAl}(110)$ surface with the magnitude of $1.24 \mu_{B}$. We realized that the spin polarization of $d_{x^{2}-y^{2}}$ and $d_{x y}$ states is the most essential in the observation of ferromagnetic ground state. We also found that the magnetic dipolar interaction is more important than the spin-orbit interaction in the study of anisotropy. We hope our results stimulate further experimental verification.

This work was supported by grant No. R01-2005-000-11001-0 from the Basic Research Program of the Korea Science \& Engineering Foundation. 


\section{References}

[1] P. Gambardella, A. Dallmeyer, K. Maiti, M.C. Malagoli, W. Eberhardt, K. Kern, and C. Carbone, Nature, 416, 301(2002).

[2] N. Nilius, T.M. Wallis, W. Ho, Science 297, 1853 (2002).

[3] P. Gambardella et al, Science 300, 1130 (2003)

[4] Jisang Hong and R.Q. Wu, Phys. Rev. B (Rapid Comm.) 67, 020406 (2003).

[5] Jisang Hong and R.Q. Wu, Phys. Rev. B (Rapid Comm.) 70, 060406 (2004).

[6] Varlei Rodrigues, Jefferson Bettini, Paulo C. Silva, and Daniel Ugarte, Phys. Rev. Lett. 91, 096801 (2003).

[7] A. Delin, E. Tosatti, and R. Weht, Phys. Rev. Lett. 92, 057201 (2003).

[8] N. Zabala, M.J. Puska, and R.M. Nieminen, Phys. Rev. Lett. 80, 3336 (1998).

[9] D.M Gillingham, I Lininghton and J.A.C Bland, J. Phys: Condens. Mater. 14 L567 (2002).

[10] D.M Gillingham, C. Müller, and J.A.C Bland, J. Appl. Phys. 95, 6995 (2004).

[11] Jisang Hong and R.Q.Wu, accepted.

[12] J.P. Perdew, K. Burke, and M. Ernzerhof, Phys. Rev. Lett. 77, 3865 (1996).

[13] Jisang Hong and R.Q. Wu, submitted.

[14] D.D. Koelling and B.N. Harmon, J. Phys. C: Solid State Phys. 10, 3107 (1977).

[15] X.D. Wang, R.Q. Wu, S.D. Wang, and A.J. Freeman, Phys. Rev. B 54, 61 (1996). 
Fig. 1. Schematic illustration of unit cell of $\mathrm{Ti} / \mathrm{NiAl}(110)$ considered in our calculations. The numbers indicate atomic type

Fig. 2. DOS of majority and minority spin electrons: (a) free standing Ti (b) $\mathrm{Ti} / \mathrm{NiAl}(110)$.

Fig. 3. m-resolved DOS of Ti: (a) free standing Ti (b) Ti/NiAl(110). 
$\mathrm{Ti}$

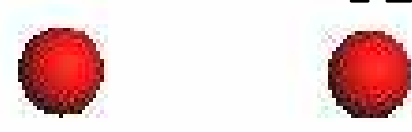

$\mathrm{Ni}$

888888

8888888

8888888

8888888

888888 

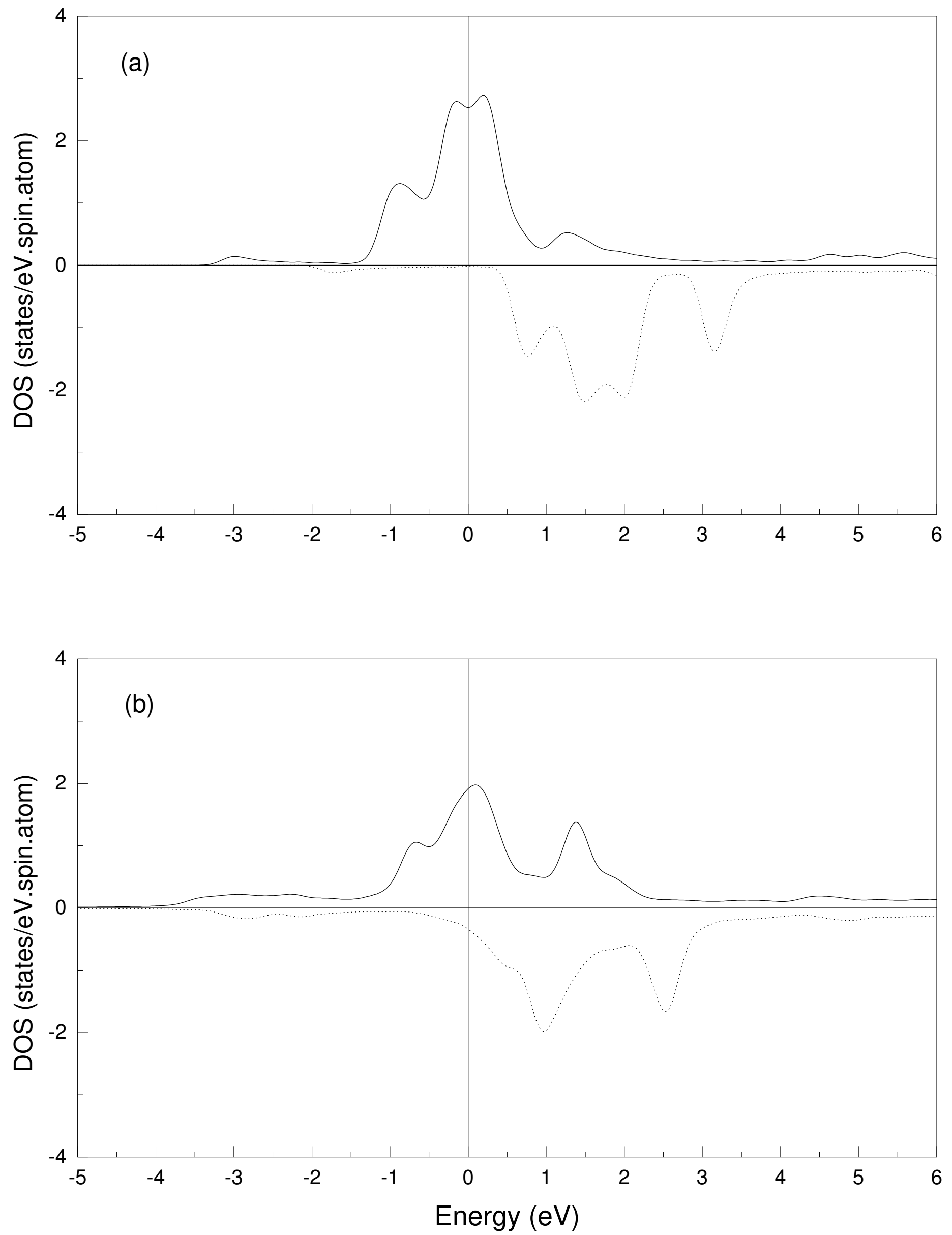

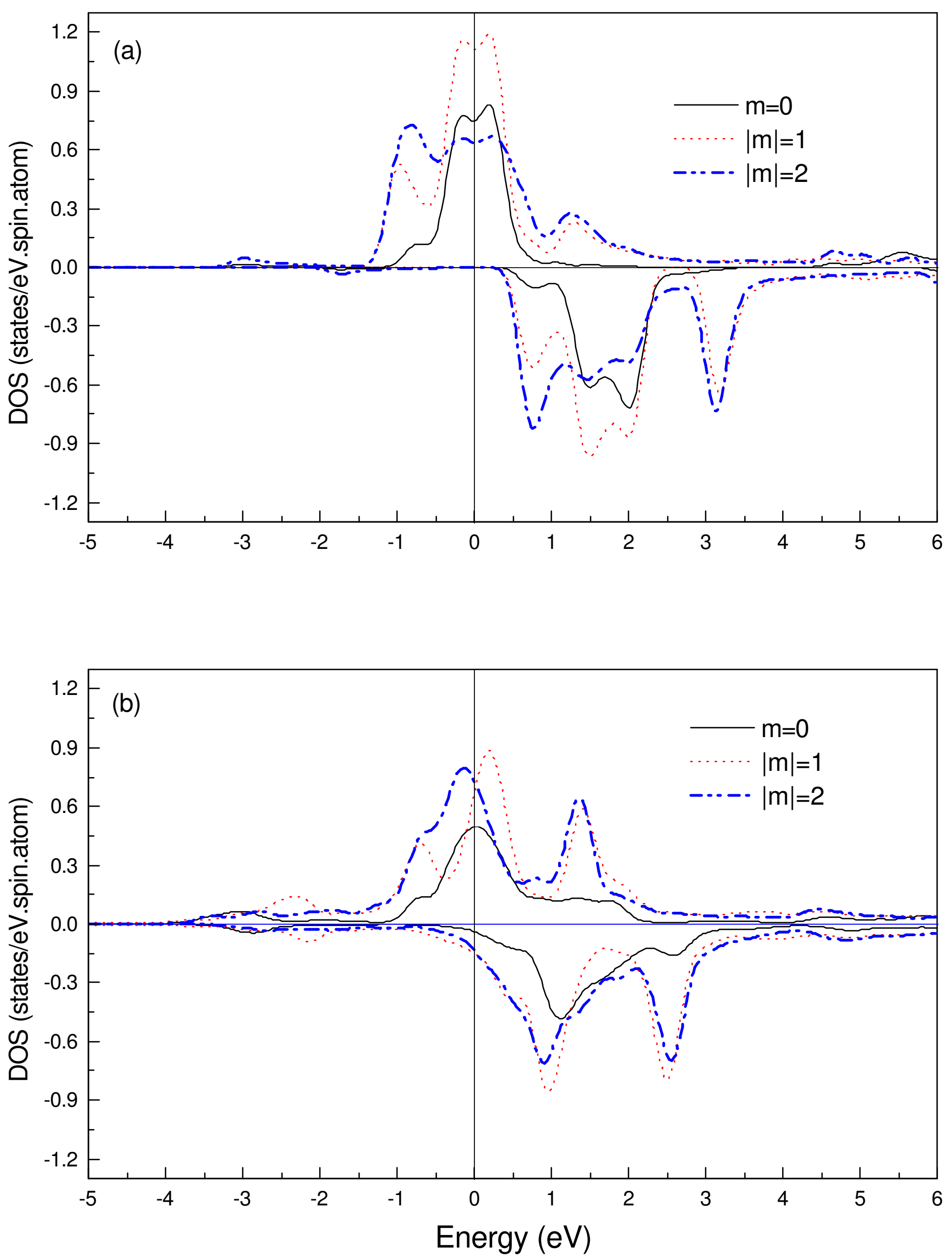